\title{
Static Hybrid Multihop Relaying
}

\author{
${ }^{1}$ Sami Touati, ${ }^{2}$ Hatem Boujemaa \\ ${ }^{1}$ King Saud University, Kingdom of Saudi Arabia \\ ${ }^{2}$ University of Carthage, Sup'Com, COSIM Laboratory, Tunisia \\ stouati@ksu.edu.sa, boujemaa.hatem@supcom.rnu.tn
}

Received: March 15, 2021. Revised: April 16, 2021. Accepted: April 19, 2021. Published: April 28, 2021.

\begin{abstract}
In this work, we propose a new static hybrid multihop relaying protocol where some relays amplify the received signal whereas the remaining ones use Decode and Forward (DF) relaying. The relaying mode in each relay is set using the distance between the different nodes or the average SNR. The exact and asymptotic bit error probabilities are derived. Simulation results are provided in different contexts to compare the performance of hybrid relaying to conventional $\mathrm{AF}$ and DF relaying.
\end{abstract}

Keywords : Cooperative diversity, Decode and Forward relaying, Amplify and Forward Relaying, Rayleigh fading channels.

\section{INTRODUCTION}

Multihop relaying can be used in wireless networks in order to increase the coverage and reduce the outage probability. By increasing the number of hops, the average Signal to Noise Ratio (SNR) at the destination increases for the same power spent by the source and all relays. Amplify and Forward (AF) and Decode and Forward (DF) relaying are the most widely used protocols. In AF relaying, each relay amplifies the received signal using an adaptive gain. DF relays demodulate the signal and regenerate it. To avoid error propagation, they transmit only if they correctly decoded. Therefore, AF protocol is less complex than DF but it may offer worse performance than DF since the noise is amplified with the useful signal.

The Bit Error Probabilities (BEP) of dual-hop and multihop relaying using AF have been derived in [1]-[3]. Multihop multibranch relaying using DF has been studied in [4]. Some adaptive $\mathrm{AF}$ and $\mathrm{DF}$ relaying protocols were proposed in the literature for dual-hop relaying [5]-[9]. In [5]-[7], the relay first tries to decode the received signal. If the decoding succeeds, it transmits the decoded signal as in DF protocol. If the decoding fails, the relay simply amplifies the received signal. In [8], the relay estimates the BEP using log-likelihood ratios. If the estimated BEP is above a given threshold, DF relaying is used. Otherwise, the relay amplifies the received signal since it contains no or only few errors. In [9], a DecodeAmplify Forward protocol is proposed where a relay amplifies the soft output of the channel decoder output. In [10], an adaptive relaying protocol has been proposed called Threshold based adaptive Decode-Amplify and Forward relaying. In this protocol, AF is used only when the instantaneous SNR is larger than the average one. Otherwise, DF is used. Static hybrid dual hop relaying has been proposed in [11]-[12] where some relays use AF and faraway relays decode the received signal. In this static dual hop hybrid relaying protocol, the relaying mode is fixed as long as the relay node position remains unchanged. The relaying mode of each relay is based on relays position or the average SNR. To the best of the authors' knowledge, static multihop hybrid relaying, where some relays use $\mathrm{AF}$ and other relays use DF relaying, hasn't been previously proposed or studied. The paper scope is to set the relaying mode (AF or DF) for each relay to have a good compromise between complexity and performance. For example, for 3 hops network, we will compare the performance of AF-DF, DF-AF, DF-DF and AF-AF. AF-DF means that the first relays amplifies the received signal whereas the next one use decode and forward relaying. ${ }^{1}$

The paper is organized as follows. The next section deals with hybrid three hops relaying whereas section III is dedicated to four hops relaying. Section IV generalizes the previous results to multihop relaying. Section V provides some simulation results. Section VI draws some conclusions and perspectives.

\section{HYBRID THREE HOPS RELAYING}

\section{A. Performance analysis of DF-DF relaying}

We consider a cooperative network composed of a source $S$, two relays $R_{1}$ and $R_{2}$, and a destination $D$. Each symbol $x$ is transmitted from $S$ to $R_{1}$, then $R_{1}$ detects it and forwards it to $R_{2}$. Finally, $R_{2}$ detects the symbol and forwards it to $D$. Both relays transmit only they have correctly detected. Otherwise, $R_{1}$ and/or $R_{2}$ are idle and the symbol will not be received at $D$.We assume that each relay perfectly judges if it has correctly decoded.

The Bit Error Probability (BEP) at D is given by

$$
P_{e, D}^{D F D F}=1-\left(1-P_{e, S R_{1}}\right)\left(1-P_{e, R_{1} R_{2}}\right)\left(1-P_{e, R_{2} D}\right)
$$

where $P_{e, X Y}$ is the BEP between nodes $X$ and $Y,(X, Y) \in$ $\left\{\left(S, R_{1}\right) ;\left(R_{1}, R_{2}\right) ;\left(R_{2}, D\right)\right\}$. For $M-Q A M$ modulation and Rayleigh fading channels, it is given by

$$
P_{e, X Y}=\frac{A}{2}\left(1-\sqrt{\frac{\bar{\Gamma}_{X Y}}{\bar{\Gamma}_{X Y}+\frac{2}{B}}}\right),
$$

$A$ and $B$ depend on the considered modulation (for example, $A=1, B=2$ for $B P S K), \bar{\Gamma}_{X Y}$ is the average $S N R$ of $X-Y$ link

$$
\bar{\Gamma}_{X Y}=E\left(\Gamma_{X Y}\right)=\frac{E_{X}}{N_{0}} E\left(\left|h_{X Y}\right|^{2}\right),
$$

$E($.$) is the expectation operator, E_{X}$ is the transmitted energy per symbol by $X$ and $E\left(\left|h_{X Y}\right|^{2}\right)$ is the power of the Rayleigh fading channel $h_{X Y}$.

\footnotetext{
${ }^{1}$ This work was supported by the Research Center of College of Computer and Information Sciences, King Saud University, under Grant No. RC130398. The authors are grateful for this support.
} 
The asymptotic BEP at D can be written as

$$
\begin{aligned}
\widetilde{P}_{e, D}^{D F D F}= & \frac{A}{2 B}\left[\frac{1}{\bar{\Gamma}_{S R_{1}}}+\frac{1}{\bar{\Gamma}_{R_{1} R_{2}}}+\frac{1}{\bar{\Gamma}_{R_{2} D}}\right] \\
& -\left(\frac{A}{2 B}\right)^{2}\left[\frac{1}{\bar{\Gamma}_{S R_{1}}}\left(\frac{1}{\bar{\Gamma}_{R_{1} R_{2}}}+\frac{1}{\bar{\Gamma}_{R_{2} D}}\right)+\frac{1}{\bar{\Gamma}_{R_{1} R_{2}}} \frac{1}{\bar{\Gamma}_{R_{2} D}}\right]
\end{aligned}
$$

D. Performance analysis of the proposed AF-DF relaying

For AF-DF relaying, the BEP at D is given by

(4)

$$
P_{e, D}=1-\left(1-P_{e, S R_{1} R_{2}}\right)\left(1-P_{e, R_{2} D}\right),
$$

\section{$B$. Performance analysis of $A F-A F$ relaying}

For AF relaying, the SNR at the destination can be written as and [2]

$\Gamma_{D}=\frac{\Gamma_{S R_{1} R_{2}} \Gamma_{R_{2} D}}{1+\Gamma_{S R_{1} R_{2}}+\Gamma_{R_{2} D}}<\Gamma_{D}^{u p}=\min \left(\Gamma_{S R_{1}}, \Gamma_{R_{1} R_{2}}, \Gamma_{R_{2} D}\right)$,

where $\Gamma_{S R_{1} R_{2}}$ is the $\mathrm{SNR}$ of $S R_{1} R_{2}$ AF link.

For Rayleigh fading channels, $\Gamma_{D}^{u p}$ follows an exponential distribution with mean

$$
\overline{\Gamma_{D}^{u p}}=\frac{1}{\frac{1}{\bar{\Gamma}_{S R_{1}}}+\frac{1}{\bar{\Gamma}_{R_{1} R_{2}}}+\frac{1}{\bar{\Gamma}_{R_{2} D}}} .
$$

We deduce a lower bound of the BEP at the destination for $M$-QAM modulations :

$P_{e, D}^{A F A F}>\int A Q(\sqrt{B \gamma}) p_{\Gamma_{D}^{u p}}(\gamma) d \gamma=\frac{A}{2}\left(1-\sqrt{\frac{\overline{\Gamma_{D}^{u p}}}{\overline{\Gamma_{D}^{u p}}+\frac{2}{B}}}\right)$

Using (7), the asymptotic BEP of AFAF relaying is given by

$$
\widetilde{P}_{e, D}^{A F A F}=\frac{A}{2 B}\left[\frac{1}{\bar{\Gamma}_{S R_{1}}}+\frac{1}{\bar{\Gamma}_{R_{1} R_{2}}}+\frac{1}{\bar{\Gamma}_{R_{2} D}}\right]
$$

\section{Performance analysis of the proposed DF-AF relaying}

In DF-AF relaying, the first relay detects the transmitted symbol whereas $R_{2}$ amplifies the received signal. The BEP at the destination is given by

$$
P_{e, D}^{D F A F}=1-\left(1-P_{e, S R_{1}}\right)\left(1-P_{e, R_{1} R_{2} D}\right),
$$

We can derive the exact expression of the BEP of $R_{1} R_{2} D$ link [3]. However, we prefer to use min upper bound to obtain closed form expressions

$$
\Gamma_{R_{1} R_{2} D}<\Gamma_{R_{1} R_{2} D}^{u p}=\min \left(\Gamma_{R_{1} R_{2}}, \Gamma_{R_{2} D}\right)
$$

This upper bound gives

$$
P_{e, R_{1} R_{2} D}>\frac{A}{2}\left[1-\sqrt{\frac{\bar{\Gamma}_{R_{1} R_{2} D}^{u p}}{\bar{\Gamma}_{R_{1} R_{2} D}^{u p}+\frac{2}{B}}}\right]
$$

where

$$
\bar{\Gamma}_{R_{1} R_{2} D}^{u p}=\frac{\bar{\Gamma}_{R_{1} R_{2}} \bar{\Gamma}_{R_{2} D}}{\bar{\Gamma}_{R_{1} R_{2}}+\bar{\Gamma}_{R_{2} D}}
$$

Combining (9) and (11), we obtain a lower bound on the BEP at the destination for hybrid DF-AF relaying.

The asymptotic BEP of hybrid DFAF relaying is given by

$$
\begin{aligned}
\widetilde{P}_{e, D}^{D F A F}= & \frac{A}{2 B}\left[\frac{1}{\bar{\Gamma}_{S R_{1}}}+\frac{1}{\bar{\Gamma}_{R_{1} R_{2}}}+\frac{1}{\bar{\Gamma}_{R_{2} D}}\right] \\
& -\left(\frac{A}{2 B}\right)^{2} \frac{1}{\bar{\Gamma}_{S R_{1}}}\left[\frac{1}{\bar{\Gamma}_{R_{1} R_{2}}}+\frac{1}{\bar{\Gamma}_{R_{2} D}}\right]
\end{aligned}
$$

$$
P_{e, S R_{1} R_{2}}>\frac{A}{2}\left[1-\sqrt{\frac{\bar{\Gamma}_{S R_{1} R_{2}}^{u p}}{\bar{\Gamma}_{S R_{1} R_{2}}^{u p}+\frac{2}{B}}}\right],
$$$$
\bar{\Gamma}_{S R_{1} R_{2}}^{u p}=\frac{\bar{\Gamma}_{S R_{1}} \bar{\Gamma}_{R_{1} R_{2}}}{\bar{\Gamma}_{S R_{1}}+\bar{\Gamma}_{R_{1} R_{2}}} .
$$

The asymptotic BEP of hybrid AFDF relaying is given by

$$
\begin{aligned}
\widetilde{P}_{e, D}^{A F D F}= & \frac{A}{2 B}\left[\frac{1}{\bar{\Gamma}_{S R_{1}}}+\frac{1}{\bar{\Gamma}_{R_{1} R_{2}}}+\frac{1}{\bar{\Gamma}_{R_{2} D}}\right] \\
& -\left(\frac{A}{2 B}\right)^{2} \frac{1}{\bar{\Gamma}_{R_{2} D}}\left[\frac{1}{\bar{\Gamma}_{S R_{1}}}+\frac{1}{\bar{\Gamma}_{R_{1} R_{2}}}\right]
\end{aligned}
$$

\section{E. Performance comparison of the different protocols}

By comparing the asymptotic BEP, we first notice that AFAF relaying offers worse performance than the other protocols. Also, DFDF offers always better performance than DFAF and AFDF. The difference between the asymptotic performance of DFAF and AFDF is given by

$$
\widetilde{P}_{e, D}^{D F A F}-\widetilde{P}_{e, D}^{A F D F}=\left(\frac{A}{2 B}\right)^{2} \frac{1}{\bar{\Gamma}_{R_{1} R_{2}}}\left[\frac{1}{\bar{\Gamma}_{R_{2} D}}-\frac{1}{\bar{\Gamma}_{S R_{1}}}\right],
$$

which clearly shows that DFAF outperforms AFDF only when $\bar{\Gamma}_{R_{2} D}>\bar{\Gamma}_{S R_{1}}$. Furthermore, equations (4) and (13) show that the proposed hybrid DFAF protocol offer the same performance as DFDF relaying for large values of $\bar{\Gamma}_{R_{1} R_{2}}$ or $\bar{\Gamma}_{R_{2} D}$ (i.e $R_{2}$ is close to $R_{1}$ or the destination). Also, equations (4) and (17) show that the proposed hybrid AFDF protocol offer the same performance as DFDF relaying for large values of $\bar{\Gamma}_{S R_{1}}$ or $\bar{\Gamma}_{R_{1} R_{2}}$ (i.e $R_{1}$ is close to $S$ or $R_{2}$ ). The proposed hybrid relaying protocols are less complex than DFDF since decoding is not performed at one relay which reduces power consumption and transmission delays.

\section{HYBRID FOUR HOPS RELAYING}

\section{A. DFDFDF relaying}

For DFDFDF relaying, the BEP at the destination is given by

$$
\begin{aligned}
P_{e, D}^{D F D F D F}= & 1-\left(1-P_{e, S R_{1}}\right)\left(1-P_{e, R_{1} R_{2}}\right) \\
& \times\left(1-P_{e, R_{2} R_{3}}\right)\left(1-P_{e, R_{3} D}\right)
\end{aligned}
$$

Therefore, the asymptotic BEP is given by

$$
\begin{gathered}
\widetilde{P}_{e, D}^{D F D F D F}=\frac{A}{2 B}\left(\frac{1}{\bar{\Gamma}_{S R_{1}}}+\frac{1}{\bar{\Gamma}_{R_{1} R_{2}}}+\frac{1}{\bar{\Gamma}_{R_{2} R_{3}}}+\frac{1}{\bar{\Gamma}_{R_{3} D}}\right) \\
-\left(\frac{A}{2 B}\right)^{2}\left[\frac{1}{\bar{\Gamma}_{S R_{1}}}\left(\frac{1}{\bar{\Gamma}_{R_{1} R_{2}}}+\frac{1}{\bar{\Gamma}_{R_{2} R_{3}}}+\frac{1}{\bar{\Gamma}_{R_{3} D}}\right)\right. \\
\left.+\frac{1}{\bar{\Gamma}_{R_{1} R_{2}}}\left(\frac{1}{\bar{\Gamma}_{R_{2} R_{3}}}+\frac{1}{\bar{\Gamma}_{R_{3} D}}\right)+\frac{1}{\bar{\Gamma}_{R_{2} R_{3}} \bar{\Gamma}_{R_{3} D}}\right]
\end{gathered}
$$




\section{B. AFAFAF relaying}

Similarly to section II.B, a lower bound of the BEP at the destination for AFAFAF relaying is given by

$$
P_{e, D}^{A F A F A F}>\frac{A}{2}\left(1-\sqrt{\frac{\overline{\Gamma_{D}^{u p}}}{\frac{2}{B}+\overline{\Gamma_{D}^{u p}}}}\right),
$$

where

$$
\frac{1}{\overline{\Gamma_{D}^{u p}}}=\frac{1}{\bar{\Gamma}_{S R_{1}}}+\frac{1}{\bar{\Gamma}_{R_{1} R_{2}}}+\frac{1}{\bar{\Gamma}_{R_{2} R_{3}}}+\frac{1}{\bar{\Gamma}_{R_{3} D}} .
$$

The asymptotic BEP is expressed as follows

$$
\widetilde{P}_{e, D}^{A F A F A F}=\frac{A}{2 B}\left(\frac{1}{\bar{\Gamma}_{S R_{1}}}+\frac{1}{\bar{\Gamma}_{R_{1} R_{2}}}+\frac{1}{\bar{\Gamma}_{R_{2} R_{3}}}+\frac{1}{\bar{\Gamma}_{R_{3} D}}\right) .
$$

\section{AFDFDF relaying}

For 4-hops AFDFDF relaying, the BEP at $D$ is given by $P_{e, D}^{A F D F D F}=1-\left(1-P_{e, S R_{1} R_{2}}\right)\left(1-P_{e, R_{2} R_{3}}\right)\left(1-P_{e, R_{3} D}\right)$.

The asymptotic BEP is given by

$$
\begin{aligned}
\widetilde{P}_{e, D}^{A F D F D F} & =\frac{A}{2 B}\left[\frac{1}{\bar{\Gamma}_{S R_{1}}}+\frac{1}{\bar{\Gamma}_{R_{1} R_{2}}}+\frac{1}{\bar{\Gamma}_{R_{2} R_{3}}}+\frac{1}{\bar{\Gamma}_{R_{3} D}}\right] \\
-\left(\frac{A}{2 B}\right)^{2} & {\left[\frac{1}{\bar{\Gamma}_{S R_{1}}}\left(\frac{1}{\bar{\Gamma}_{R_{2} R_{3}}}+\frac{1}{\bar{\Gamma}_{R_{3} D}}\right)+\frac{1}{\bar{\Gamma}_{R_{2} R_{3}} \bar{\Gamma}_{R_{3} D}}\right.} \\
& \left.+\frac{1}{\bar{\Gamma}_{R_{1} R_{2}}}\left(\frac{1}{\bar{\Gamma}_{R_{2} R_{3}}}+\frac{1}{\bar{\Gamma}_{R_{3} D}}\right)\right] .
\end{aligned}
$$

Therefore, the difference between the asymptotic BEP of AFDFDF and DFDFDF relaying is

$$
\widetilde{P}_{e, D}^{A F D F D F}-\widetilde{P}_{e, D}^{D F D F D F}=\left(\frac{A}{2 B}\right)^{2} \frac{1}{\bar{\Gamma}_{S R_{1}} \bar{\Gamma}_{R_{1} R_{2}}}
$$

AFDFDF relaying offers close performance to DFDFDF relaying if $R_{1}$ is close to $S$ or $R_{2}$. We also notice that the difference between the asymptotic BEP of AFDFDF and DFDFDF is proportional to the product of the inverse SNRs of the AF relaying link (i.e. $\frac{1}{\bar{\Gamma}_{S R_{1}} \overline{\bar{\Gamma}}_{R_{1} R_{2}}}$ ).

\section{DFAFDF relaying}

For DFAFDF relaying, the BEP at $D$ is given by $P_{e, D}^{D F A F D F}=1-\left(1-P_{e, S R_{1}}\right)\left(1-P_{e, R_{1} R_{2} R_{3}}\right)\left(1-P_{e, R_{3} D}\right)$.

The asymptotic BEP is given by

$$
\begin{aligned}
& \widetilde{P}_{e, D}^{D F A F D F}=\frac{A}{2 B}\left[\frac{1}{\bar{\Gamma}_{S R_{1}}}+\frac{1}{\bar{\Gamma}_{R_{1} R_{2}}}+\frac{1}{\bar{\Gamma}_{R_{2} R_{3}}}+\frac{1}{\bar{\Gamma}_{R_{3} D}}\right] \\
&-\left(\frac{A}{2 B}\right)^{2}\left[\frac{1}{\bar{\Gamma}_{S R_{1}}}\left(\frac{1}{\bar{\Gamma}_{R_{1} R_{2}}}+\frac{1}{\bar{\Gamma}_{R_{2} R_{3}}}+\frac{1}{\bar{\Gamma}_{R_{3} D}}\right)\right. \\
&\left.+\frac{1}{\bar{\Gamma}_{R_{3} D}}\left(\frac{1}{\bar{\Gamma}_{R_{1} R_{2}}}+\frac{1}{\bar{\Gamma}_{R_{2} R_{3}}}\right)\right] .
\end{aligned}
$$

Therefore, the difference between the asymptotic BEP of DFAFDF and DFDFDF relaying is given by

$$
\widetilde{P}_{e, D}^{D F A F D F}-\widetilde{P}_{e, D}^{D F D F D F}=\left(\frac{A}{2 B}\right)^{2} \frac{1}{\bar{\Gamma}_{R_{1} R_{2}} \bar{\Gamma}_{R_{2} R_{3}}}
$$

DFAFDF relaying offers close performance to DFDFDF relaying if $R_{2}$ is close to $R_{1}$ or $R_{3}$. We also notice that (29) is proportional to the product of the inverse SNRs of the AF relaying link (i.e. $\frac{1}{\bar{\Gamma}_{R_{1} R_{2}} \bar{\Gamma}_{R_{2} R_{3}}}$ ).

\section{E. DFDFAF relaying}

For DFDFAF relaying, the BEP at $D$ is given by $P_{e, D}^{D F D F A F}=1-\left(1-P_{e, S R_{1}}\right)\left(1-P_{e, R_{1} R_{2}}\right)\left(1-P_{e, R_{2} R_{3} D}\right)$.

The asymptotic BEP is given by

$$
\begin{gathered}
\widetilde{P}_{e, D}^{D F D F A F}=\frac{A}{2 B}\left[\frac{1}{\bar{\Gamma}_{S R_{1}}}+\frac{1}{\bar{\Gamma}_{R_{1} R_{2}}}+\frac{1}{\overline{\bar{\Gamma}}_{R_{2} R_{3}}}+\frac{1}{\bar{\Gamma}_{R_{3} D}}\right] \\
-\left(\frac{A}{2 B}\right)^{2}\left[\frac{1}{\bar{\Gamma}_{S R_{1}}}\left(\frac{1}{\bar{\Gamma}_{R_{1} R_{2}}}+\frac{1}{\bar{\Gamma}_{R_{2} R_{3}}}+\frac{1}{\bar{\Gamma}_{R_{3} D}}\right)\right. \\
\left.+\frac{1}{\bar{\Gamma}_{R_{1} R_{2}}}\left(\frac{1}{\bar{\Gamma}_{R_{2} R_{3}}}+\frac{1}{\bar{\Gamma}_{R_{3} D}}\right)\right] .
\end{gathered}
$$

Therefore, the difference between the asymptotic BEP of DFDFAF and DFDFDF relaying is given by

$$
\widetilde{P}_{e, D}^{D F D F A F}-\widetilde{P}_{e, D}^{D F D F D F}=\left(\frac{A}{2 B}\right)^{2} \frac{1}{\bar{\Gamma}_{R_{2} R_{3}} \bar{\Gamma}_{R_{3} D}}
$$

DFDFAF relaying offers close performance to DFDFDF relaying if $R_{3}$ is close to $R_{2}$ or $D$. We also notice that (32) is proportional to the product of the inverse SNRs of the AF relaying link (i.e. $\frac{1}{\bar{\Gamma}_{R_{2} R_{3}} \bar{\Gamma}_{R_{3} D}}$ ).

\section{F. AFDFAF relaying}

For AFDFAF relaying, the BEP at $D$ is given by

$$
P_{e, D}^{A F D F A F}=1-\left(1-P_{e, S R_{1} R_{2}}\right)\left(1-P_{e, R_{2} R_{3} D}\right) .
$$

The asymptotic BEP is given by

$$
\begin{aligned}
\widetilde{P}_{e, D}^{A F D F A F}= & \frac{A}{2 B}\left[\frac{1}{\bar{\Gamma}_{S R_{1}}}+\frac{1}{\bar{\Gamma}_{R_{1} R_{2}}}+\frac{1}{\bar{\Gamma}_{R_{2} R_{3}}}+\frac{1}{\bar{\Gamma}_{R_{3} D}}\right] \\
& -\left(\frac{A}{2 B}\right)^{2}\left[\frac{1}{\bar{\Gamma}_{S R_{1}}}\left(\frac{1}{\bar{\Gamma}_{R_{2} R_{3}}}+\frac{1}{\bar{\Gamma}_{R_{3} D}}\right)\right. \\
& \left.+\frac{1}{\bar{\Gamma}_{R_{1} R_{2}}}\left(\frac{1}{\bar{\Gamma}_{R_{2} R_{3}}}+\frac{1}{\bar{\Gamma}_{R_{3} D}}\right)\right]
\end{aligned}
$$

Therefore, the difference between the asymptotic BEP of AFDFAF and DFDFDF relaying is given by

$\widetilde{P}_{e, D}^{A F D F A F}-\widetilde{P}_{e, D}^{D F D F D F}=\left(\frac{A}{2 B}\right)^{2}\left[\frac{1}{\bar{\Gamma}_{S R_{1}} \bar{\Gamma}_{R_{1} R_{2}}}+\frac{1}{\bar{\Gamma}_{R_{2} R_{3}} \bar{\Gamma}_{R_{3} D} D}\right]$

AFDFAF relaying offers close performance to DFDFDF relaying if $R_{1}$ is close to $S$ or $R_{2}$ and $R_{3}$ is close to $R_{2}$ or $D$. We also notice that (35) is proportional to the sum of the product of the inverse SNRs of the two AF relaying link $\left(S R_{1} R_{2}\right.$ and $R_{2} R_{3} D$ i.e. $\frac{1}{\bar{\Gamma}_{S R_{1}} \overline{\bar{\Gamma}}_{R_{1} R_{2}}}$ and $\left.\frac{1}{\bar{\Gamma}_{R_{2} R_{3}} \bar{\Gamma}_{R_{3} D} D}\right)$. The last equation, (26) and (29) shows that AFDFAF relaying offers worse performance than AFDFDF and DFAFDF relaying. 


\section{G. AFAFDF relaying}

For AFAFDF relaying, the BEP at $D$ is given by

$$
P_{e, D}^{A F A F D F}=1-\left(1-P_{e, S R_{1} R_{2} R_{3}}\right)\left(1-P_{e, R_{3} D}\right) .
$$

The asymptotic BEP is given by

$$
\begin{gathered}
\widetilde{P}_{e, D}^{A F A F D F}=\frac{A}{2 B}\left[\frac{1}{\overline{\bar{\Gamma}}_{S R_{1}}}+\frac{1}{\overline{\bar{\Gamma}}_{R_{1} R_{2}}}+\frac{1}{\overline{\bar{\Gamma}}_{R_{2} R_{3}}}+\frac{1}{\overline{\bar{\Gamma}}_{R_{3} D}}\right] \\
-\left(\frac{A}{2 B}\right)^{2} \frac{1}{\overline{\bar{\Gamma}}_{R_{3} D}}\left[\frac{1}{\overline{\bar{\Gamma}}_{S R_{1}}}+\frac{1}{\overline{\bar{\Gamma}}_{R_{1} R_{2}}}+\frac{1}{\overline{\bar{\Gamma}}_{R_{2} R_{3}}}\right]
\end{gathered}
$$

Therefore, the difference between the asymptotic BEP of AFAFDF and DFDFDF relaying is given by

$$
\begin{aligned}
\widetilde{P}_{e, D}^{A F A F D F}-\widetilde{P}_{e, D}^{D F D F D F}= & \left(\frac{A}{2 B}\right)^{2}\left[\frac{1}{\bar{\Gamma}_{S R_{1}} \bar{\Gamma}_{R_{1} R_{2}}}\right. \\
& \left.+\frac{1}{\bar{\Gamma}_{R_{2} R_{3}}}\left(\frac{1}{\bar{\Gamma}_{S R_{1}}}+\frac{1}{\bar{\Gamma}_{R_{1} R_{2}}}\right)\right]
\end{aligned}
$$

AFAFDF relaying offers close performance to DFDFDF if $R_{1}$ is close to $S$ or $R_{2}$ and $R_{2}$ is close to $R_{3}$. The last equation, (26) and (29) shows that AFAFDF relaying offers worse performance than AFDFDF and DFAFDF relaying.

\section{H. DFAFAF relaying}

For DFAFAF relaying, the BEP at $D$ is given by

$$
P_{e, D}^{D F A F A F}=1-\left(1-P_{e, S R_{1}}\right)\left(1-P_{e, R_{1} R_{2} R_{3} D}\right) .
$$

The asymptotic BEP is given by

$$
\begin{aligned}
\widetilde{P}_{e, D}^{D F A F A F}= & \frac{A}{2 B}\left[\frac{1}{\bar{\Gamma}_{S R_{1}}}+\frac{1}{\bar{\Gamma}_{R_{1} R_{2}}}+\frac{1}{\bar{\Gamma}_{R_{2} R_{3}}}+\frac{1}{\bar{\Gamma}_{R_{3} D}}\right] \\
& -\left(\frac{A}{2 B}\right)^{2} \frac{1}{\bar{\Gamma}_{S R_{1}}}\left[\frac{1}{\bar{\Gamma}_{R_{1} R_{2}}}+\frac{1}{\bar{\Gamma}_{R_{2} R_{3}}}+\frac{1}{\bar{\Gamma}_{R_{3} D}}\right]
\end{aligned}
$$
(40)performance as DFDF (see equations (4) and (17)) and 1.1 $\mathrm{dB}$ gain with respect to AFAF for $\mathrm{BER}=0.05$.

Fig. 2 shows the BER at $\mathrm{D}$ for three hops relaying when $d_{S R_{1}}=d_{R_{1} R_{2}}=1$ and $d_{R_{2} D}=0.4$. We notice that DFAF relaying outperforms AFDF which was expected since $\bar{\Gamma}_{S R_{1}}<$ Therefore, the difference between the asymptotic BEP of DFAFAF and DFDFDF relaying is given by

$$
\begin{aligned}
\widetilde{P}_{e, D}^{D F A F A F}-\widetilde{P}_{e, D}^{D F D F D F}= & \left(\frac{A}{2 B}\right)^{2}\left[\frac{1}{\bar{\Gamma}_{R_{1} R_{2}} \bar{\Gamma}_{R_{2} R_{3}}}\right. \\
& \left.+\frac{1}{\bar{\Gamma}_{R_{3} D}}\left(\frac{1}{\bar{\Gamma}_{R_{1} R_{2}}}+\frac{1}{\bar{\Gamma}_{R_{2} R_{3}}}\right)\right]
\end{aligned}
$$

$$
\bar{\Gamma}_{R_{2} D} \text {. DFAF offers the same performance as DFDF relaying }
$$
and $1.1 \mathrm{~dB}$ gain with respect to AFAF for BER=0.05.

Fig. 3 shows the BER at D for four hops relaying when $d_{S R_{1}}=1, d_{R_{1} R_{2}}=d_{R_{2} R_{3}}=2$ and $d_{R_{3} D}=0.2$. Since $R_{3}$ is close to D, DFDFAF offers close performance to DFDFDF (see equation (32)). DFDFAF offers $1 \mathrm{~dB}$ gain with respect to AFAFAF and $3 \mathrm{~dB}$ gain with respect to noncooperative communications $\left(d_{S D}=d_{S R_{1}}+d_{R_{1} R_{2}}+d_{R_{2} R_{3}}+d_{R_{3} D}\right)$. The order in performance of the different hybrid protocols is in accordance with the analysis provided in section III. Besides, AFDFDF offers the same performance as AFDFAF with is confirmed by the theoretical analysis (see (25) and (34)). DFAFDF offers the same performance as DFAFAF (see (28) and (40)). AFAFDF offers the same performance as AFAFAF (see (23) and (37)).

\section{CONCLUSIONS}

In this paper, we have proposed and analyzed the performance of hybrid multihop relaying where some relays amplify the received signal and the remaining ones use DF relaying. We have shown that hybrid relaying offers better performance than AF relaying and close performance to DF relaying. The proposed protocol is less complex than DF and reduces power consumption and transmission delays since decoding is not performed at all nodes. 


\section{REFERENCES}

[1] P. A. Anghel, M. Kaveh, "Exact symbol error probability of a cooperative network in a Rayleigh fading environnement", IEEE Trans. on Wireless Commun., Vol. 3, no 5, pp. 1416-1421, Sept. 2004.

[2] M. O. Hasna, M. S. Alouini, "End-to-end performance of transmission systems with relays over rayleigh fading channels", IEEE Trans. Wireless Commun., Vol. 2, pp. 1126-1131, Nov. 2003.

[3] F. Yilmaz, O. Kucur, M. S. Alouini, "A Novel Framework on Exact Average Symbol Error Probabilities of multihop transmission over Amplifyand-Forward Relay fading channels", IEEE ISWCS, 2010.

[4] S. Amara, H. Boujemaa, "Multihop multibrach DF relaying for cooperative systems", IEEE Vehicular Technology Conference Spring, Budapest, May 2011.

[5] M. R. Souryal, B. R. Vojcic, "Performance of Amplify-and-Forward and Decode-and-Forward Relaying in Rayleigh Fading with Turbo Codes", ICASSP, Vol. 4, pp. 681-684, May 2006.

[6] T. Q. Duong, H. J. Zepernick, "On the Performance Gain of Hybrid Decode-Amplify-Forward Cooperative Communications", EURASIP Journal on Wireless Communications and Networking, Volume 2009, 2009.

[7] Q. Li, S. H. Ting, A. Pandharipande, Y. Han, "Adaptive Two-Way Relaying and Outage Analysis", IEEE Transactions on Wireless Communications, Vol. 8, Issue 6, pp. 3288-3299, June 2009.

[8] J. C. Fricke, M. M. Butt, P. A. Hoeher, "Quality-Oriented Adaptive Forwarding for Wireless Relaying", IEEE Communications Letters, Vol. 12, No. 3, pp. 200-201, June 2009.

[9] X. Bao, J. T. Li, "Efficient Message Relaying for Wireless User Cooperation: Decode-Amplify-Forward (DAF) and Hybrid DAF and CodedCooperation", IEEE Transactions on Wireless Communications, Vol. 6, Issue 11, pp. 3975-3984, November 2007.

[10] S. Bouanen, H. Boujemaa, W. Ajib, "Threshold based adaptive decode amplify forward relaying protocol for cooperative systems", IEEE Wireless and Mobile Computing Conference, pp. 725-730, Istanbul, July 2011.

[11] H. Boujemaa, "Static hybrid amplify and forward (AF) and decode and forward (DF) relaying for cooperative systems", Elsevier, Physical communications, pp. 196-205, vol. 4, 2011.

[12] M. Ben Said, H. Boujemaa, "Static hybrid amplify decode and forward cooperative ARQ with opportunistic relaying, " IEEE Intern. Conference on Commun., Computing and Control Applications, Hammamet, March 2010.

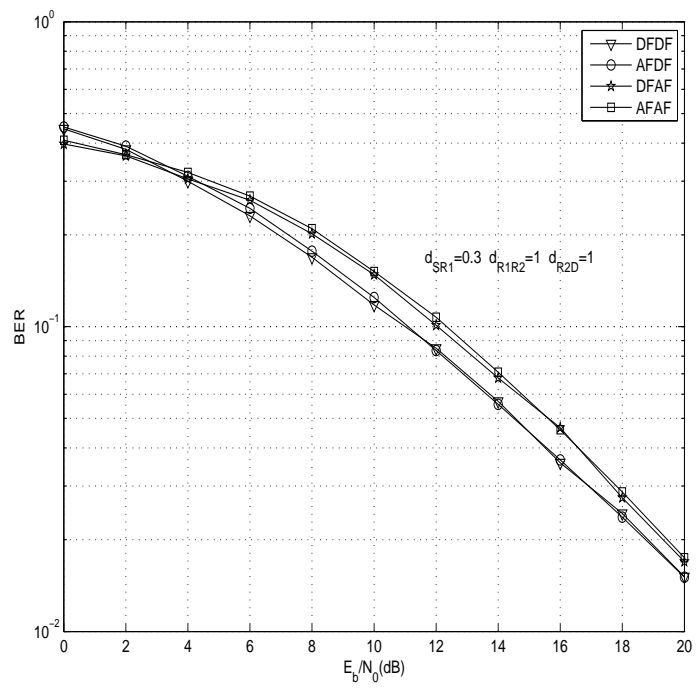

Fig. 1. BEP of 3-hops relaying with respect average SNR : $d_{S R_{1}}=0.3$, $d_{R_{1} R_{2}}=1$ and $d_{R_{2} D}=1$.

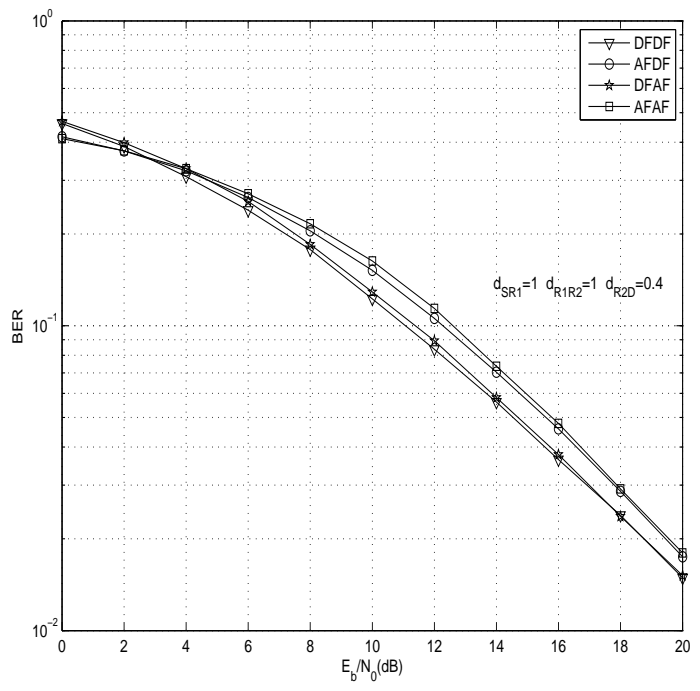

Fig. 2. BEP of hybrid 3-hops relaying with respect average SNR : $d_{S R_{1}}=1$, $d_{R_{1} R_{2}}=1$ and $d_{R_{2} D}=0.4$.

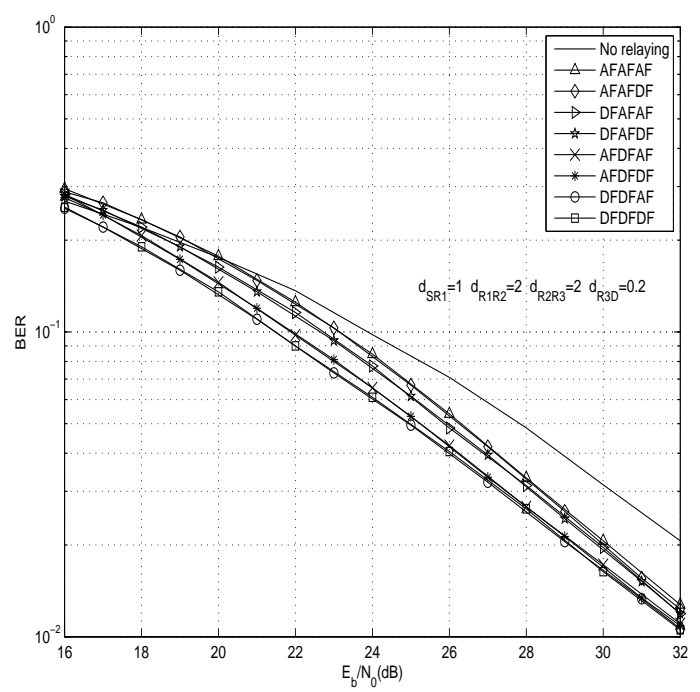

Fig. 3. BEP of hybrid 4-hops relaying with respect average SNR : $d_{S R_{1}}=1$, $d_{R_{1} R_{2}}=2, d_{R_{2} R_{3}}=2$ and $d_{R_{3} D}=0.2$.

\section{Creative Commons Attribution License 4.0 (Attribution 4.0 International, CC BY 4.0)}

This article is published under the terms of the Creative Commons Attribution License 4.0

https://creativecommons.org/licenses/by/4.0/deed.en_US 